\title{
Design and Simulation of Ear and Tongue Based Biometrics for Attendance Management System
}

Adebayo, O.Y., Adigun, A.A., Adeyemo, T.T. and Isola, E.O.

Abstract: Multimodal biometrics has engrossed a lot of interest in recent years as it offers a more dependable scheme for verification and authentication. Multimodal biometrics includes a combination of information from different biometric modalities. This research work simulates a novel method with a view of attendance management using ear and tongue images. Principal Component Analysis was employed for feature extraction of the biometric modalities and Self Organizing Feature Map was used for training and testing of the system. The method was evaluated using six thousand ear images and four thousand tongue images. The acquired images were preprocessed and features extracted were fused at this stage. The fusion results of ear and tongue images demonstrated better performance for attendance management.

Keywords: Multimodal, Biometrics, Attendance Management, PCA, SOFM, Fusion

\section{Introduction}

Biometrics is the safest method to meet the digitization necessities of identity and virtualization in the field of information society. It refers to the automated authentication of a person through the use of physiological or behavioural traits [1]. Practically, no modality is best as each modality has its own merits and demerits. Biometric systems are used in authentication to reduce financial fraud and increase security in various fields. In recent time, ear recognition is drawing more and more consideration. Ear recognition has turn out to be a proficient and alluring strategy [2]. It is rigidly set in the middle of the side of the head and does not undergo a significant change with time, unlike the face. The ear does not undergo changes in facial expression and is rigidly set in the middle of the side of the head

\footnotetext{
Adebayo, O.Y., Adigun, A.A. and Isola, E.O. (Dept. of Information and Communication Technology, Osun State University, Osogbo, Osun-State, Nigeria)

Adeyemo, T.T. (Dept. of Information and Communication Technology, Elizade University, Ilara Mokin, Ondo State)

Corresponding Author: olajide.adebayo@uniosun.edu.ng

Telephone Number:+2348035234019

Submitted: $17-$ Sept-2020

Accepted: 08-Feb-2021
}

so that the immediate background is knowable [3].

Multimodal biometric systems have proved to be better off unimodal biometric systems in term of performance. The features from several biometric modalities are fused either in the feature extraction stage or in the matching stage of the process. [4]. Managing student attendance engrosses keeping track of attendance of the student in the classroom. Attendance management is vital because attendance affects students academic performance. Several modalities such as iris [5], face [6], Radio Frequency Identification [7], Clustering-based [8], Deep Learning [9], image processing and machine learning [10] and fingerprint [11] have been employed by researchers to manage presence.

[8] Presented an attendance management system based on an unsupervised learning approach using signature. The system is in three phases; data pre-processing, extraction of relevant features and verification phases. When the system was tested to predict students' average attendance for a given course, 0.96 was the mean square error obtained which demonstrated the effectiveness of the clustering technique and 
showed that the technique can be effective when deployed for managing attendance. The future direction is to make provision for a scheme that detect and reject overlapping signatures.

[10] Proposed an image and machine learningbased attendance monitoring system for classrooms. Contrasting the traditional manual marking of attendance which can mark fake attendance; the proposed system detects faces from the classroom and mark attendance accordingly by feature extraction and matching using machine learning techniques and the performance was evaluated using Convolution Neural Network algorithm. The results presented showed that the faces of students present in the classroom were accurately detected. [12] developed a system for automatic attendance capturing which incorporated two different deep learning algorithms; convolution neural network face detection algorithm and, SeetaFace face recognition algorithm. In order to overcome limitations associated with low-resolution image, the work employed $4 \mathrm{~K}$ HD video for face detection and, recognition. Using the system to conduct various experiments, different results obtained revealed that the system achieved five variations of classroom attendance; total absence, late arrival, early departure, free access, and carelessness. They concluded those small classrooms of $6 \mathrm{~m}$ or less requires a $1080 \mathrm{P}$ video camera while a bigger classroom of $9 \mathrm{~m}$ requires a $4 \mathrm{~K}$ surveillance video camera for attendance management.

[13] Presented a different approach based on ear and tongue biometric modalities for access control. Principal component analysis technique was deployed to extract different features from images of ear and tongue obtained and, training with testing of the system was done using SOFM. Three thousand (3000) ear and, two thousand (2000) tongue images were used in testing and evaluation of the system. The primary source of those images was through a camera and was later fused at the feature extraction phase. Obtained results showed an improved accuracy of $99.73 \%$ and, a huge step in the right direction for user access control. In future, the extracted features could be optimized for optimal and better performance.

Given the above-identified limitations, this paper introduced an ear and tongue multimodal based technique for attendance management by incorporating collaborative authentication.

\section{Materials and Methods}

This section presents the research methodology employed to realize the research aim and objective. The first step was the ear and tongue image acquisition (see fig. 1a-d and $2 \mathrm{a}-\mathrm{d}$ ), the second stage implements the preprocessing procedure by removing the noise from the ear and tongue images and minimizing the distortion introduced in the acquired ear and tongue images. Features were extracted from the preprocessed images and fused at the feature extraction stage. The performance of the attendance management system was evaluated based on Recognition Accuracy and Equal Error Rate (EER).

\section{A. Ear and Tongue Image Acquisition}

The data that were used in this research work were acquired using a digital camera. A programming interface was developed to acquire 6,000 ears and 4,000 tongue images of 1000 subjects or volunteers (i.e. $1000 * 6$ for Ear and 1000* 4 Tongue $=10,000)$. All these images were stored and used as training and testing datasets. 


\section{B. Data Preprocessing}

The following preprocessing steps were carried out in this research work:

\section{Step 1: Resizing of Tongue and Ear Images}

The acquired images were resized from the default dimension of $480 \times 640$ to $200 \times 200$ pixels.

\section{Step 2: Extraction of Region of Interest (ROI)}

A tongue and ear ROI extracting scheme must be extremely effective and efficient. As depicted in Figure 1, five stages were followed in the extraction of ROI of resized ear and tongue images adopted from the work of Alwan and Alani [14]. The stages are as follows:

i. A median filter will be used to denoise the ear and tongue images.

ii. Binarizing the ear and tongue images by using the Ostu thresholding algorithm.

iii. Apply edge detection algorithm to detect the boundary.

iv. Determine two important reference points and rotate the image

v. Draw rectangle or square around the ROI

\section{Step 3: Histogram Equalization (HE)}

Histogram Equalization (HE) is a technique used to enhance the quality of images for achieving more image information. $\mathrm{HE}$ was used to enhancing only these intensity values in the region of interest of ear and tongue images. The histogram of an image as proposed by [15] with gray levels in the range $[0, \mathrm{~L}-1]$ is a discrete function $q(r)=n_{r}$, where $\mathrm{r}$ is the gray level and $n_{r}$ is the number of pixels in the image having gray level $r$. Using $\mathrm{HE}$ for ear and tongue image normalization as depicted in equations 1 and 3, histogram equalization is accomplished for certain intensity band between lower limit $b$ and upper limit c. $\mathrm{T}(\mathrm{r})$ is cumulative distribution function in the intensity range between $b$ and $c$ and is given by

$$
T(r)=\sum_{k=b}^{r} z(k), \quad b \leq r \leq c .(1)
$$

The normalized distribution function is;

$$
\mathrm{T}_{\mathrm{n}}(\mathrm{r})=\mathrm{T}(\mathrm{r}) / \mathrm{T}(\mathrm{c})
$$

where $T(c)$ is the maximum value of the cumulative distribution function in the selected intensity band. The normalized values can be scaled between $\mathrm{b}$ and $\mathrm{c}$ as follows;

$\bar{T}(r)=\operatorname{int}\left[\frac{T_{n}(r)-T_{n}(b)}{1-T_{n}(b)} *(c-b)+b\right], b \leq r \leq$

$$
c \text {, }
$$

where $T_{n}(b)$ is the minimum value in the cumulative probability density function vector $T_{n}(r) . \bar{T}(r)$ is the transformed value for each gray level in the selected intensity band.

\section{Extraction of Ear and Tongue Features}

As presented in equations 4 to 9 , the feature set in the preprocessed images was extracted using Principal Component Analysis (PCA) as illustrated below;

$$
A^{I I}=\frac{1}{A^{I I}} \quad \sum_{i=1}^{A^{I I}} x_{i}
$$

where:

$x_{i}=$ dimensional vector

$m_{j}=$ mean centred image

$\mathrm{C}=$ Covariance

$$
\begin{aligned}
& m_{j}=x_{i}-A^{I I} \\
& C=A^{I I} A^{I I T}
\end{aligned}
$$

assuming is eigenvector and $n_{i}$ is the eigenvalue:

Projecting $L \lambda A^{I I}$

$$
A^{I I} A^{I I T}=\lambda\left(A^{I I} n\right)
$$

$$
\begin{aligned}
& \Omega^{\mathrm{II}}=\left\{\mathrm{n}_{1}, \mathrm{n}_{2} \ldots \ldots \ldots \mathrm{n}_{\mathrm{i}}\right\}^{\mathrm{T}} \\
& E_{k}^{I I}=\left\|\Omega^{I I}-\Omega_{k}^{I I}\right\|
\end{aligned}
$$


Finally, minimum unidentified data is assigned to $\mathrm{K}$

\section{Fusion}

The two feature vectors, a feature vector extracted from the tongue and second represents the feature vector extracted from the ear. These two feature vectors are combined and fused to present a novel user authentication system. The fusion of feature vectors of tongue and ear is given below:

$\mathrm{X}_{1}=$ Tongue feature vector

$\mathrm{Y}_{1}=$ Ear feature vector

$\mathrm{M}=$ Fusion value

$\mathrm{M}=\mathrm{X}_{1}+\mathrm{Y}_{1} \quad$ (see Fig. 4)

\section{Results and Discussion}

The code implementing the attendance management system was tested using $M A T L A B \quad 7.7 .0 \quad$ (R2016a) on Windows 7 Ultimate 64-bit operating system, $A M D$ Atblon (tm) X2 DualCore QL-66 central processing unit with a speed of $2.2 G H Z, 4 G B$ random access memory and 500GB HHD. The proposed multimodal based attendance management system was evaluated on a data set of 10,000 pairs of tongue and ear images of 1000 subjects. The training and testing data set contains 6,000 ear and tongue images and 4,000 ear and tongue images. The performance of the system was evaluated based on Recognition Accuracy and Equal Error Rate (EER). It is impeccable that the performance of the proposed multimodal method (Ear and Tongue) for attendance management is better than unimodal biometric as depicted in Fig 5. The projected method in managing the attendance of people has a $99.90 \%$ performance recognition and a $0.003 \%$ error rate than appraised methods for attendance management.

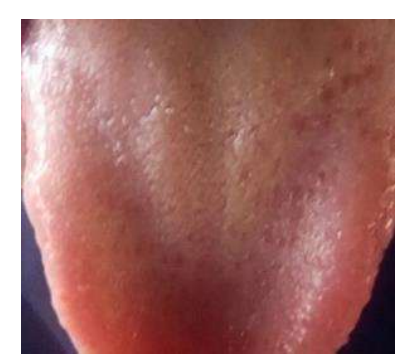

a - Acquired Tongue Image

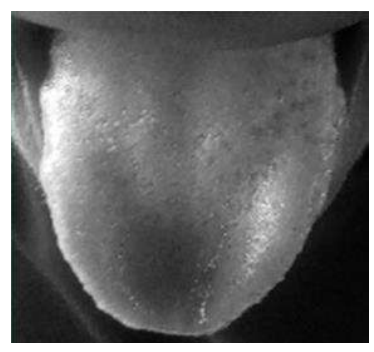

b - Cropped Tongue

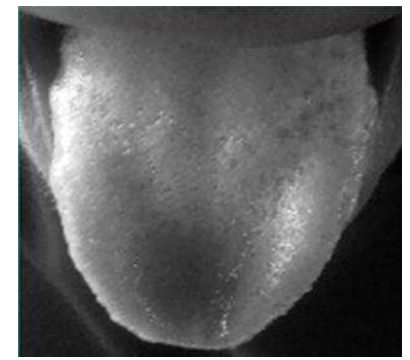

c - Preprocessed Tongue Image

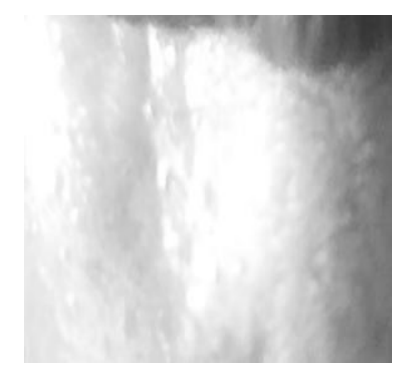

\section{d - Extracted Tongue Feature}

Figure 1: Tongue Image Preprocessing and Feature Extraction 


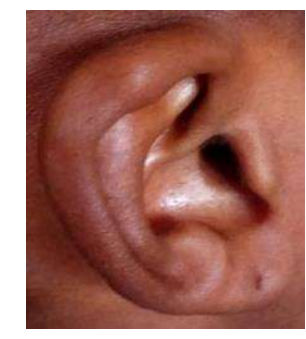

a - Acquired Ear Image

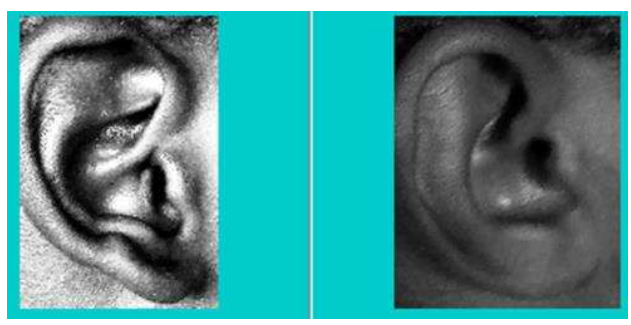

b - Cropped Ear Image

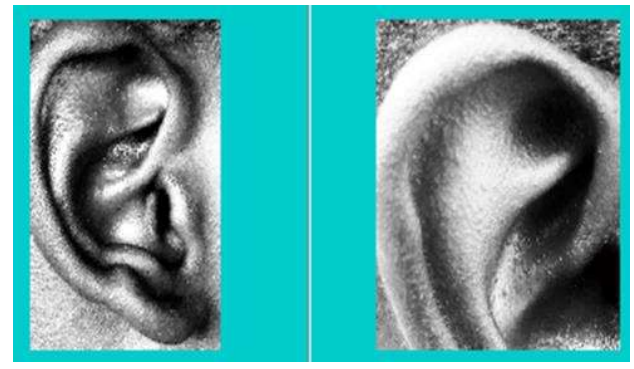

c - Preprocessed Ear Image

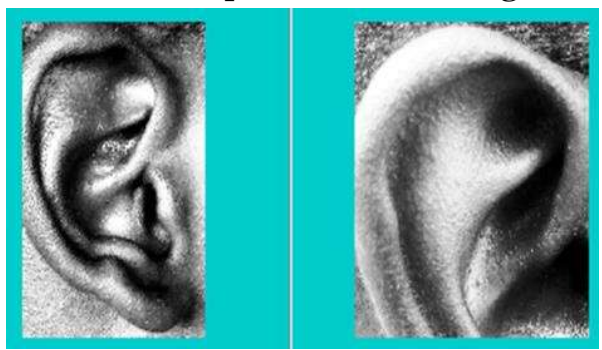

d - Extracted Ear Feature

Figure 2: Ear Image Processing and Feature Extraction

\section{Conclusion}

In this work, a multimodal biometric authentication system was developed which uses ear and tongue features for attendance management as indicated in Figure 5. The research investigated the reliability of the developed method and performance of multimodal biometric systems. The

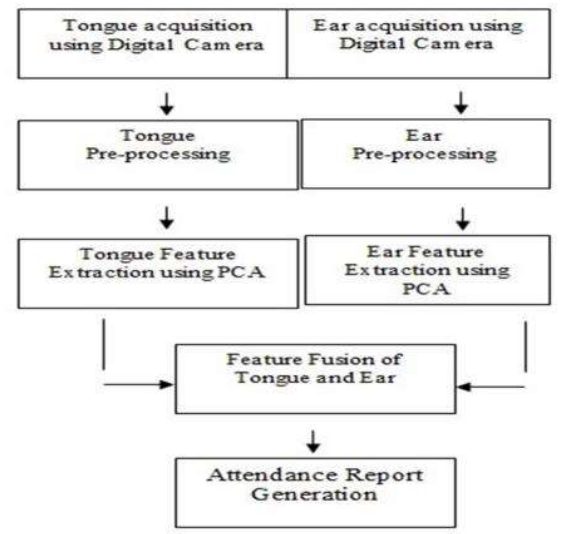

Figure 3: Block Diagram of the Developed Multimodal Attendance Management System

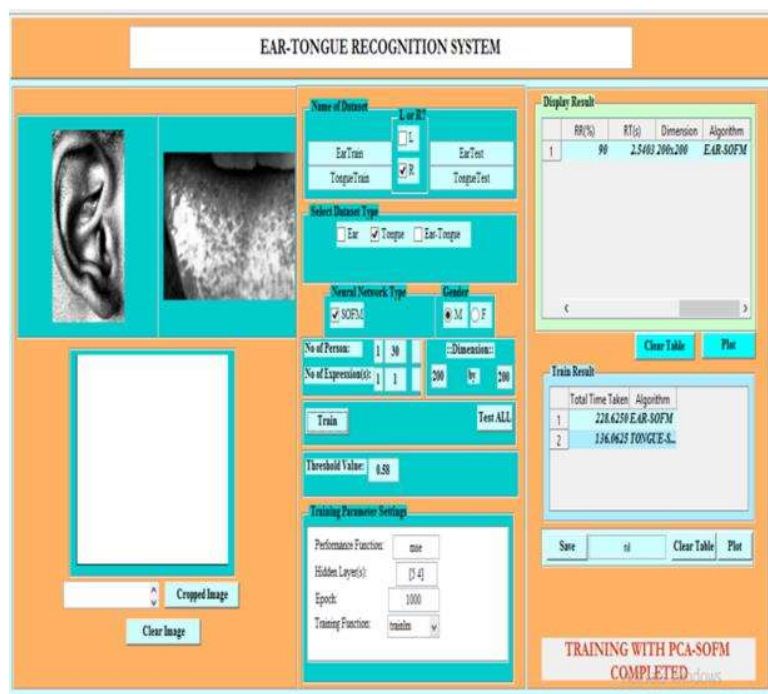

Figure 4: Fusion of Ear and Tongue Features in MATLAB Environment

\begin{tabular}{|c|c|c|c|c|c|}
\hline & \multicolumn{2}{|c|}{ Choose List: A } & Attendances List & v & Clear List \\
\hline & 1 & 2 & 3 & & \\
\hline 1 & 1111 & 3:31:08 PM & Registered & & \\
\hline 2 & 8332 & 10:57:38 AM & Registered & & \\
\hline 3 & 1010 & 11:04:57 AM & Registered & & \\
\hline 4 & 1200 & 11:59:39 AMI & Registered & & \\
\hline 5 & 1990 & 12:18.25 PIA & Registered & & \\
\hline 6 & 5555 & 2:46:21 PM & Not Register & & \\
\hline 7 & 6666 & 2.53:19 PM & Registered & & \\
\hline 8 & 3000 & 3:12:14 PII & Registered & & \\
\hline 9 & 3000 & 3:13:10 PIII & Registered & & \\
\hline 10 & 3000 & 3:13:53 PM & Registered & & \\
\hline 11 & 1111 & 3:15:06 PM & Not Register & & \\
\hline 12 & 3000 & 3:15:42 PII & Registered & & \\
\hline 13 & 1010 & 3:16:39 PMI & Not Register & & \\
\hline 14 & 8332 & 3:17:29 PM & Not Register & & \\
\hline 15 & 1200 & 3:18:19 PM & Not Register & & \\
\hline
\end{tabular}

Figure 5: Attendance List in Multimodal Attendance Management System. 
experimental results have revealed that a multimodal biometric authentication system is much more reliable and might be used in realtime attendance management systems. The developed method has higher performance and low error rates other than appraised multimodal methods. Future work should take account of learning the effect of some optimization algorithms on the performance of the projected multimodal approach and the taking on more biometric traits.

\section{References}

[1] Jain, A.K., Bolle, R. and Pankanti, S. "Biometrics: Personal Identification in Networked Society", Kluwer Academic Publishers, 2001.

[2] Down, M.P. and Sands, R.J. "Biometrics: An Overview of the Technology, Challenges and Control Considerations", Information Systems Control Journal, vol. 4, 2004, pp. 53-56.

[3] Hiew, M.S., Hishammuddin, A., Rohayanti, H. and Razib, M.O. "Multimodal Biometrics: Weighted Score Level Fusion Based on Non-Ideal Iris and Face Images", Expert Systems with Applications, vol. 41, no. 11, 2014, pp. 5390-5404.

[4] Gaganpreet, K. and Dheerendra, S. "A Novel Biometric System based on Hybrid Fusion Speech, Signature and Tongue", International Journal of Computer Applications; vol. 119, no. 7, 2015, pp. 30-39.

[5] Kadry, S. and Smaili, M. (2010). "Wireless Attendance Management System Based on Iris Recognition", Scientific Research and Essays, vol. 5, no. 12, 2010, pp. 1428-1435.

[6] Balcoh, N.K., Yousaf, M.H., Ahmad, W. and Baig, M.I. "Algorithm for Efficient Attendance Management: Face Recognition Based Approach", International Journal of Computer Science Issues, vol. 9, no. 4, 2012, pp. 146-150.

[7] Arulogun, O.T., Olatunbosun, A., Fakolujo, O.A. and Olaniyi, O.M. "RFID-Based Student's Attendance Management System", International Journal of Scientific and Engineering Research, vol. 4, no. 2, 2013, pp. 1-9.
[8] Laruba, A. and Hamza, O.S. "A Clustering-based Offline Signature Verification System for Managing Lecture Attendance", Information Technology and Computer Science, vol. 9, no. 7, 2017, pp. 51-60.

[9] Dan, W., Rong, F. and Zuying, L. "Classroom Attendance Auto-management Based on Deep Learning Advances in Social Science", Proceedings of 2nd International Conference on Education, Sports, Arts and Management Engineering, Paris France, 2017, pp. 1523 - 1528.

[10] Kirtiraj, K., Manasi, J., Shivam, M. and Tushar, I. "Attendance Monitoring System Using Image Processing and Machine Learning", International Journal of Advance Engineering and Research Development Special Issue on Recent Trends in Data Engineering, vol. 4, no. 5 (Special Issue), 2017, pp. 1-3.

[11] Sifatnur Rahman, Mahabur Rahman, Md Mijanur Rahman "Automated Student Attendance System using Fingerprint Recognition", Edelweiss Appli Sci Tech vol. 2, no. 1, 2018, pp. 90-94.

[12] Dan, W., Rong, F. and Zuying, L. "Classroom Attendance Auto-management Based on Deep Learning, Advances in Social Science", Proceedings of 2nd International Conference on Education, Sports, Arts and Management Engineering, Paris France, 2017, pp. $1523-1528$.

[13] Adebayo Y.O., Adigun, A.A. and Isola, E.O. "Ear and Tongue Based Multimodal Access Control System", Anale. Seria Informatică., vol. XVII, no. fasc. 2, 2019, pp. 85-92.

[14] Alwan, L.J. and Alani, H.A. "Region of Interest Extraction of Palm Vein Image", International Journal of Scientific and Engineering Research, vol. 7, no. 12, 2016, pp. 850-854.

[15] Mohammed, S.S, Nabila, E., Fathi, F. and Ghada, K.G. "Band-Limited Histogram Equalization for Mammograms Contrast Enhancement", International Biomedical Engineering Conference, Cairo, Giza, 2012, pp. 154-15 